\title{
Comparisons of the Different Frequencies of Input Data for Neural Networks in Foreign Exchange Rates Forecasting
}

\author{
Wei Huang ${ }^{1,2}$, Lean $\mathrm{Yu}^{2}$, Shouyang Wang ${ }^{2}$, Yukun $\mathrm{Bao}^{1}$, and Lin Wang ${ }^{1}$ \\ ${ }^{1}$ School of Management, Huazhong University of Science and Technology, \\ WuHan, 430074, China \\ \{yukunbao, wanglin\}@mail.hust.edu.cn \\ ${ }^{2}$ Institute of Systems Science, Academy of Mathematics and Systems Sciences, \\ Chinese Academy of Sciences, Beijing, 100080, China \\ \{whuang, yulean, sywang\} @amss.ac.cn
}

\begin{abstract}
We compare the predication performance of neural networks with the different frequencies of input data, namely daily data, weekly data, monthly data. In the 1 day and 1 week ahead prediction of foreign exchange rates forecasting, the neural networks with the weekly input data performs better than the random walk models. In the 1 month ahead prediction of foreign exchange rates forecasting, only the special neural networks with weekly input data perform better than the random walk models. Because the weekly data contain the appropriate fluctuation information of foreign exchange rates, it can balance the noise of daily data and losing information of monthly data.
\end{abstract}

\section{Introduction}

Exchange rates are one of the most important economic indices in the international monetary markets. Because of its complicated nonlinear behavior, many researchers employ neural networks to forecast foreign exchange rates. Several design factors significantly affect the prediction performance of neural networks [1]. Although foreign exchange rates are high-frequency financial time series, fluctuating every minute, the researchers seldom employ the hourly observations for forecasting. Because there is too much noises in the observations of high-frequency, no body use the frequency that is higher than daily data. It is a common practice to predict the future value daily ahead with daily data[2,3], to predict the future value weekly ahead with weekly data[4-6], to predict the future value monthly ahead with monthly data[7-10]. Hann and Steurer compare the prediction performances of neural network models with linear monetary models in forecasting USD/DEM by using the monthly data and weekly data. Out-of-sample results show that, for weekly data, neural networks are much better than linear models and a random walk model. However, if monthly data are used, neural networks do not show much improvement over linear models[11].

In fact, different frequencies of input data may affect the performances of neural networks, due to the volatility of currency movements. However, few researchers investigate the effect of different frequencies of input data. Our contribution of the 
paper is to compare the prediction performance of the neural networks by using the different frequencies of input data. The remainder of this paper is organized as follows. Section 2 gives the experiment design. Section 3 discusses the experiment results. Finally, conclusions are given in Section 4.

\section{Experiment Design}

We employ the three frequencies of data, namely daily data $\left\{y_{t}^{d}\right\}$, weekly data $\left\{y_{t}^{w}\right\}$, monthly data $\left\{y_{t}^{m}\right\}$. The daily data is the closing price of every trading day. The weekly data is compiled from the average of daily data in that week. The monthly data is compiled from the average of daily data in that month.

\subsection{Random Walk Models}

The weak form of efficient market theory describes that prices always fully reflect the available information, that is, a price is determined by the previous value in the time series because all the relevant information is summarized in that previous value. An extension of this theory is the random walk (RW) model. The random walk model uses the actual value of current period to predict the future value of next period as follows:

$$
\hat{y}_{t+1}=y_{t}
$$

where $\hat{y}_{t+1}$ is the predicted value of the next period; $y_{t}$ is the actual values of current period.

Therefore, the predicted values of next day, week, month by RW model are computed in the following way:

$$
\begin{aligned}
& \hat{y}_{t+1}^{d}=y_{t}^{d} \\
& \hat{y}_{t+1}^{w}=y_{t}^{w} \\
& \hat{y}_{t+1}^{m}=y_{t}^{m}
\end{aligned}
$$

\subsection{Neural Network Models}

In this study, we employ one of the widely used neural networks models, the threelayers back-propagation neural network (BPNN), for foreign exchange rates forecasting. The activation function used for all hidden nodes is the logistic function, while the linear function is employed in the output node. The number of input nodes is a very important factor in neural network analysis of a time series since it corresponds to the number of past lagged observations related to future values. To avoid introducing a bias in results, we choose the number of input nodes as 3,5, 7 and 9, respectively. Because neural networks with one input nodes are too simple to capture the 
complex relationships between input and output, and it is rarely seen in the literatures that the number of input nodes is more than nine. Generally speaking, too many nodes in the hidden layer produce a network that memorizes the input data and lacks the ability to generalize. Parsimony is a principle for designing neural networks. Hence, the number of hidden nodes is equal to the number of input nodes.

Because there is no daily data of economic indicators such exportation, importation, we employ univariate input for the neural networks. The inputs of neural network are the past, lagged observations of exchange rates; the output is the future value. In some sense, the neural networks of univariate input are equivalent to a nonlinear autoregressive mode as follows:

$$
\hat{y}_{t+1}=F\left(y_{t}, y_{t-1}, \ldots, y_{t-K}\right)
$$

where $\hat{y}_{t+1}$ is the output of the neural networks, namely the predicted value of the next period; $y_{t}, y_{t-1}, \ldots, y_{t-K}$ are the inputs for the neural networks, namely the actual value at the period $t, t-1, \ldots, t-K$, respectively; function $F$ is a nonlinear function determined by the neural networks; $K$ is the max lag period, which is determined by the number of input nodes. In the study, when the number of input nodes is $3,5,7$ and $9, K$ is $2,4,6,8$, respectively.

We employ daily data, weekly data, monthly data as inputs of the neural networks to predict the future foreign exchange rates of the next day as follows:

$$
\begin{aligned}
& \hat{y}_{t+1}^{d}=F_{d}^{d}\left(y_{t}^{d}, y_{t-1}^{d}, \ldots, y_{t-K}^{d}\right) \\
& \hat{y}_{t+1}^{d}=F_{d}^{w}\left(y_{t}^{w}, y_{t-1}^{w}, \ldots, y_{t-K}^{w}\right) \\
& \hat{y}_{t+1}^{d}=F_{d}^{m}\left(y_{t}^{m}, y_{t-1}^{m}, \ldots, y_{t-K}^{m}\right)
\end{aligned}
$$

where $\hat{y}_{t+1}^{d}$ is the output of the neural networks, namely the predicted value of the next day; function $F_{d}^{d}, F_{d}^{w}, F_{d}^{m}$ are the nonlinear functions determined by the neural networks with the input of daily data, weekly data, monthly data, respectively.

We employ daily data, weekly data, monthly data as inputs of the neural networks to predict the future foreign exchange rates of the next week as follows:

$$
\begin{aligned}
& \hat{y}_{t+1}^{w}=F_{w}^{d}\left(y_{t}^{d}, y_{t-1}^{d}, \ldots, y_{t-K}^{d}\right) \\
& \hat{y}_{t+1}^{w}=F_{w}^{w}\left(y_{t}^{w}, y_{t-1}^{w}, \ldots, y_{t-K}^{w}\right) \\
& \hat{y}_{t+1}^{w}=F_{w}^{m}\left(y_{t}^{m}, y_{t-1}^{m}, \ldots, y_{t-K}^{m}\right)
\end{aligned}
$$

where $\hat{y}_{t+1}^{w}$ is the output of the neural networks, namely the predicted value of the next week; function $F_{w}^{d}, F_{w}^{w}, F_{w}^{m}$ are the nonlinear functions determined by the neural networks with the input of daily data, weekly data, monthly data, respectively. 
We employ daily data, weekly data, monthly data as inputs of the neural networks to predict the future foreign exchange rate of the next month as follows:

$$
\begin{aligned}
& \hat{y}_{t+1}^{m}=F_{m}^{d}\left(y_{t}^{d}, y_{t-1}^{d}, \ldots, y_{t-K}^{d}\right) \\
& \hat{y}_{t+1}^{m}=F_{m}^{w}\left(y_{t}^{w}, y_{t-1}^{w}, \ldots, y_{t-K}^{w}\right) \\
& \hat{y}_{t+1}^{m}=F_{m}^{m}\left(y_{t}^{m}, y_{t-1}^{m}, \ldots, y_{t-K}^{m}\right)
\end{aligned}
$$

where $\hat{y}_{t+1}^{m}$ is the output of the neural networks, namely the predicted value of the next month; function $F_{m}^{d}, F_{m}^{w}, F_{m}^{m}$ are the nonlinear functions determined by the neural networks with the input of daily data, weekly data, monthly data, respectively.

\subsection{Performance Measure}

We employ root of mean squared error (RMSE) to evaluate the prediction performance of neural networks as follows:

$$
\operatorname{RMSE}=\sqrt{\frac{\sum_{t}\left(y_{t}-\hat{y}_{t}\right)^{2}}{T}}
$$

where $y_{t}$ is the actual value; $\hat{y}_{t}$ is the predicted value; $T$ is the number of the predictions

\subsection{Data Preparation}

From Pacific Exchange Rate Service provided by Professor Werner Antweiler, University of British Columbia, Canada, we obtain 3291 daily observations, 678 weekly data and 156 monthly data of U.S. dollar against the British Pound (GBP) and Japanese Yen (JPY) covering the period the period from Jan 1990 to Dec, 2002. First, we produce the testing sets for each neural network models by selecting 60 patterns of the latest periods from the three datasets, respectively. Then, we produce the appropriate training sets for each neural networks model from the corresponding left data in the three datasets by using the method in [12].

\section{Experiments Results}

Table 1 shows the prediction performances of the random walk models, which are used as benchmarks of prediction performance of foreign exchange rates for the different forecasting horizons. The prediction performance become worse as the forecasting horizon becomes longer. This pattern is consistent with the assumption of random walk model.

Table 2-5 show the 1 day ahead prediction performance of the neural networks with $3,5,7,9$ input nodes, respectively. In the 1 day ahead prediction of foreign ex- 
change rates, the neural networks with weekly input data perform better than the random walk models; the neural networks with daily, monthly input data perform worse than the random walk models. Because the daily input data contain too much noise, while the monthly input data lose too much fluctuation information of foreign exchange rates at the scale of day.

Table 6-9 show the 1 week ahead prediction performance of the neural networks with 3, 5, 7, 9 input nodes, respectively. In the 1 week ahead prediction of foreign exchange rates, the neural networks with weekly input data perform better than the random walk models; the neural networks with daily, monthly input data perform worse than the random walk models. Because the daily input data can not cover the enough period which contains the behavior of foreign exchange rate at the scale of week, while the monthly input data lose some fluctuation information of foreign exchange rates at the scale of week.

Table 10-13 show the 1 month ahead prediction performance of the neural networks with $3,5,7,9$ input nodes, respectively. In the 1 month ahead prediction of foreign exchange rates, the neural networks with weekly input data perform little better than the random walk models when the number of the input nodes is 5 and 7; the other neural networks models perform worse than the random walk models. It indicates that the neural networks models are not suitable for the long term forecasting when the foreign exchange rates fluctuate a lot

Table 1. The prediction performance of the random walk models

\begin{tabular}{ccc}
\hline Forecasting horizon & RMSE of GBP & RMSE of JPY \\
\hline 1 day ahead & 0.0054715 & 0.007508 \\
1 week ahead & 0.0128701 & 0.01608 \\
1 month ahead & 0.0387545 & 0.042368 \\
\hline
\end{tabular}

Table 2. The 1 day ahead prediction performance of the neural networks with 3 input nodes

\begin{tabular}{crr}
\hline Frequency of input data & RMSE of GBP & RMSE of JPY \\
\hline daily & 0.0186794 & 0.021576 \\
weekly & 0.0054705 & 0.007439 \\
monthly & 0.0381506 & 0.041369 \\
\hline
\end{tabular}

Table 3. The 1 day ahead prediction performance of the neural networks with 5 input nodes

\begin{tabular}{ccc}
\hline Frequency of input data & RMSE of GBP & RMSE of JPY \\
\hline daily & 0.0155469 & 0.017623 \\
weekly & 0.004491 & 0.00635 \\
monthly & 0.0380584 & 0.040172 \\
\hline
\end{tabular}

Table 4. The 1 day ahead prediction performance of the neural networks with 7 input nodes

\begin{tabular}{ccc}
\hline Frequency of input data & RMSE of GBP & RMSE of JPY \\
\hline daily & 0.0149991 & 0.017417 \\
weekly & 0.004496 & 0.00636 \\
monthly & 0.0380457 & 0.039505 \\
\hline
\end{tabular}


Table 5. The 1 day ahead prediction performance of the neural networks with 9 input nodes

\begin{tabular}{ccc}
\hline Frequency of input data & RMSE of GBP & RMSE of JPY \\
\hline daily & 0.0186669 & 0.021558 \\
weekly & 0.0054671 & 0.007293 \\
monthly & 0.038303 & 0.04163 \\
\hline
\end{tabular}

Table 6. The 1 week ahead prediction performance of the neural networks with 3 input nodes

\begin{tabular}{ccc}
\hline Frequency of input data & RMSE of GBP & RMSE of JPY \\
\hline daily & 0.0187851 & 0.022372 \\
weekly & 0.012474 & 0.015306 \\
monthly & 0.0346265 & 0.034005 \\
\hline
\end{tabular}

Table 7. The 1 week ahead prediction performance of the neural networks with 5 input nodes

\begin{tabular}{ccc}
\hline Frequency of input data & RMSE of GBP & RMSE of JPY \\
\hline daily & 0.0179622 & 0.019123 \\
weekly & 0.01154 & 0.01421 \\
monthly & 0.0345032 & 0.033285 \\
\hline
\end{tabular}

Table 8. The 1 week ahead prediction performance of the neural networks with 7 input nodes

\begin{tabular}{ccc}
\hline Frequency of input data & RMSE of GBP & RMSE of JPY \\
\hline daily & 0.0172154 & 0.019116 \\
weekly & 0.011508 & 0.0143 \\
monthly & 0.0336946 & 0.033712 \\
\hline
\end{tabular}

Table 9. The 1 week ahead prediction performance of the neural networks with 9 input nodes

\begin{tabular}{ccc}
\hline Frequency of input data & RMSE of GBP & RMSE of JPY \\
\hline daily & 0.018782 & 0.022365 \\
weekly & 0.012528 & 0.015432 \\
monthly & 0.035721 & 0.035353 \\
\hline
\end{tabular}

Table 10. The 1 month ahead prediction performance of the neural networks with 3 input nodes

\begin{tabular}{crc}
\hline Frequency of input data & RMSE of GBP & RMSE of JPY \\
\hline daily & 0.0585355 & 0.063101 \\
weekly & 0.0402285 & 0.043588 \\
monthly & 0.0552075 & 0.059799 \\
\hline
\end{tabular}

According to the above results, we may see that weekly data is the appropriate frequency which matches the scale of fluctuation behavior of foreign exchange rates fluctuation behavior. Weekly data balance the noise of daily data and losing information of monthly data. 
We notice that the networks with 5,7 inputs nodes perform better than the networks with 3, 9 input nodes. Because the neural networks with 5, 7 input nodes are at the appropriate level of complexity, which balances the over-fitting and under-fitting.

Table 11. The 1 month ahead prediction performance of the neural networks with 5 input nodes

\begin{tabular}{crr}
\hline Frequency of input data & RMSE of GBP & RMSE of JPY \\
\hline daily & 0.0574925 & 0.061378 \\
weekly & 0.0383246 & 0.041754 \\
monthly & 0.0543704 & 0.058285 \\
\hline
\end{tabular}

Table 12. The 1 month ahead prediction performance of the neural networks with 7 input nodes

\begin{tabular}{ccc}
\hline Frequency of input data & RMSE of GBP & RMSE of JPY \\
\hline daily & 0.0571355 & 0.060978 \\
weekly & 0.0385933 & 0.041929 \\
monthly & 0.0540548 & 0.05809 \\
\hline
\end{tabular}

Table 13. The 1 month ahead prediction performance of the neural networks with 9 input nodes

\begin{tabular}{ccc}
\hline Frequency of input data & RMSE of GBP & RMSE of JPY \\
\hline daily & 0.0593892 & 0.062386 \\
weekly & 0.0405666 & 0.044135 \\
monthly & 0.0558715 & 0.060805 \\
\hline
\end{tabular}

\section{Conclusions}

In this paper, we investigate the effects of different frequencies of input data of foreign exchange rates forecasting with neural networks. The neural networks with the weekly input data performs better than those neural networks with the input of daily data and monthly data. Weekly data is the appropriate frequency which matches the scale of fluctuation behavior of foreign exchange rates fluctuation behavior.

\section{Acknowledgements}

This work is partially supported by National Natural Science Foundation of China (NSFC No. 70221001, 70401015) and the Key Research Institute of Humanities and Social Sciences in Hubei Province-Research Center of Modern Information Management.

\section{References}

1. Huang, W., Lai, K.K., Nakamori, Y. \& Wang, S.Y.. Forecasting foreign exchange rates with artificial neural networks: a review. International Journal of Information Technology \& Decision Making, 3(2004) 145-165

2. Walczak, S.: An empirical analysis of data requirements for financial forecasting with neural networks. Journal of Management Information System, 17(2001) 203-222 
3. Yu, L.A., Wang, S.Y., Lai, K.K.: Adaptive smoothing neural networks in foreign exchange rate forecasting. Lecture Notes in Computer Science,Vol. 3516, Springer-Verlag Berlin Heidelberg (2005) 523 - 530

4. Yao, J.T. \& Tan, C.L.: A case study on using neural networks to perform technical forecasting of forex. Neurocomputing, 34(2000) 79-98

5. Zhang, G.P. \& Berardi, V.L.: Time series forecasting with neural network ensembles: an application for exchange rate prediction. Journal of the Operational Research Society, 52(2001) 652-664

6. Zhang, G. \& Hu, M.Y.: Neural network forecasting of the British Pound/US Dollar exchange rate. Journal of Management Science, 26(1998) 495-506

7. Leung, M.T., Chen, A.S. \& Dauk, H.: Forecasting exchange rate using general regression neural networks. Computer \& Operations Research, 27(2000) 1093-1110

8. Lisi, F. \& Schiavo, R.A.: A comparison between neural networks and chaotic models for exchange rate prediction. Computational Statistical \& Data Analysis, 30(1999) 87-102

9. Qi, M. and Zhang, G.: An investigation of model selection criteria for neural network time series forecasting. European Journal of Operational Research, 132(2001) 666-680

10. Yu, L.A., Wang, S.Y., Lai, K.K.: A novel nonlinear ensemble forecasting model incorporating GLAR and ANN for foreign exchange rates. Computers and Operations Research, 32 (2005) 2523-2541

11. Hann, T.H. and Steurer, E.: Much ado about nothing? Exchange rate forecasting: Neural networks vs. linear models using monthly and weekly data. Neurocomputing, 10(1996) 323-339

12. Huang, W., Nakamori, Y., Wang, S.Y. \& Zhang, H.: Select the size of training set for financial forecasting with neural networks. Lecture Notes in Computer Science, Vol. 3497, Springer-Verlag Berlin Heidelberg (2005) 879-884 